\title{
FES99: A Global Tide Finite Element Solution Assimilating Tide Gauge and Altimetric Information
}

\author{
F. Lefèvre, ${ }^{*}$ F. H. Lyard, AND C. Le Provost \\ Laboratoire d'Etudes en Géophysique et Océanographie Spatiales, Toulouse, France \\ E. J. O. SCHRAMA \\ Faculty of Geodesy, Delft University of Technology, Delft, Netherlands
}

(Manuscript received 30 October 2001, in final form 14 February 2002)

\begin{abstract}
An improved version of the global hydrodynamic tide solutions [finite element solutions (FESs) FES94, FES95.2.1, and FES98] has been developed, implemented, and validated. The new model is based on the resolution of the tidal barotropic equations on a global finite element grid without any open boundary condition, which leads to solutions independent of in situ data (no open boundary conditions and no assimilation). The accuracy of these "free" solutions is improved by assimilating tide gauge and TOPEX/Poseidon (T/P) altimeter information through a representer assimilation method. This leads to the FES99 version of this model. For the eight main constituents of the tidal spectrum $\left(\mathrm{M}_{2}, \mathrm{~S}_{2}, \mathrm{~N}_{2}, \mathrm{~K}_{2}, 2 \mathrm{~N}_{2}, \mathrm{~K}_{1}, \mathrm{O}_{1}\right.$, and $\left.\mathrm{Q}_{1}\right)$, about 700 tide gauges and $687 \mathrm{~T} / \mathrm{P}$ altimetric measurements are assimilated. An original algorithm is developed to calculate the tidal harmonic constituents at crossover points of the T/P altimeter database. Additional work is performed for the $\mathrm{S}_{2}$ wave by reconsidering the inverse barometer correction. To complete the spectrum, 19 minor constituents have been added by admittance. The accuracy of FES99 is evaluated against the former FESs. First, it is compared to two tide gauge datasets: ST95 (95 open-ocean measurements) and ST739 (739 coastal measurements). For ST95, the root-sum square of the differences between observations and solutions is reduced from 2.8 (FES95.2.1) to $2.4 \mathrm{~cm}$ (FES99), a gain of $17 \%$ in overall accuracy. Second, the variance of the sea surface variability is calculated and compared for FES95.2.1, FES98, and FES99 at the T/P and the European Remote Sensing Satellite $(E R S-2)$ crossover data points. FES 99 performed best, with a residual standard deviation for the independent ERS-2 dataset of $13.5 \mathrm{~cm}(15.2 \mathrm{~cm}$ for FES95.2.1). Third, tidal predictions are implemented for the FESs to provide along-track estimates of the sea surface variability for T/P and ERS-2. Compared to ERS-2, FES 99 residuals are $11.8 \mathrm{~cm}(12.4 \mathrm{~cm}$ for FES95.2.1). All the accuracy tests show that FES99 is a significant improvement compared to former FESs both in the deep ocean and along coasts.
\end{abstract}

\section{Introduction}

At the present time, three global finite element solutions (FESs) are available for the scientific community and for engineering applications: FES94 (Le Provost et al. 1994), FES95.2.1 (Le Provost et al. 1998), and FES98 (Lefèvre et al. 2000). The associated models are based on a finite element approach, which allows one to solve the hydrodynamic equations on a refined discretization of the global ocean where needed, in particular, in shallow water. They provide the main constituents of the tidal spectrum. FES94 was the first of these solutions. Due to the computational constraints and the

* Current affiliation: CLS, Ramonville Saint Agne, France.

Corresponding author address: Dr. F. Lefèvre, CLS, 8-10, rue Hermés, Parc Technologique du Canal, Ramonville Saint-Agne cedex 31526, France.

E-mail: fabien.lefevre@cls.fr size of the global mesh, the hydrodynamic equations were solved by splitting the problem into subdomains, setting open boundary conditions with tide gauge information, and merging the results to provide the global solutions for the main constituents. With the optimization of the open boundary conditions by tide gauge measurements, a type of data assimilation (Lyard and Genco 1994), this solution was a real improvement, especially in shallow water. Several empirical tide models [for instance CSR3.0 (Eanes and Bettadpur 1996), AG95.1 (Andersen 1995), SR95.1 (Schrama and Ray 1994), or more recently GOT99.2 (Ray 1999)] have used FES94 as an a priori solution to compute corrections and obtain new improved solutions. But, due to the limitations of the hydrodynamic model, the uncertainties in the bathymetry or the subgrid-scale parameterization, FES94 needed to be upgraded in the deep ocean. Thus, FES95.2.1 was produced to overcome these uncertainties by assimilating an altimeter-derived dataset in the finite element hydrodynamic model. The 
assimilated dataset was extracted from the earlier empirical TOPEX/Poseidon (T/P) CSR2.0 tide solution (Eanes and Bettadpur 1994) using a simplified representer method based on a global inverse problem (Egbert et al. 1994). The set used in the assimilation was obtained from a sampling of CSR2.0 on a $5^{\circ} \times 5^{\circ}$ grid for ocean depths greater than $1000 \mathrm{~m}$. The accuracy of FES95.2.1 was improved compared to FES94. Considered as the best hydrodynamic model, FES95.2.1 was selected by the scientific community as one of the two models used for tidal corrections for the T/P altimeter with CSR3.0 and as the only correction for the European Remote Sensing Satellite (ERS-1/2) altimeter (Shum et al. 1997). But a major issue still remained: the global solutions were computed for each subdomain and merged afterward. While the tide elevation was continuous across basin limits, this was not the case for the tide velocities deduced from the spatial gradients of tidal elevations.

For four years, FES94 and FES95.2.1 have shown their qualities and defects. To get a more physical tide modeling, the noncontinuity introduced by the subdomain resolution had to be fixed and the production of FES98 marked a new step forward. By applying a block resolution method (Lyard 1999), the undesirable boundary effects were eliminated to produce a purely hydrodynamic solution independent of any in situ tide gauge data. Of course, these purely hydrodynamic global solutions are not as accurate as the empirical solutions (such as CSR3.0 or SR95.1) or as FES94 and FES95.2.1. Tide gauge information was assimilated to yield with FES98, a solution independent of altimetry. An unexpectedly good global tide solution in deep ocean was produced, although only by assimilating coastal tide gauges.

We present in this paper a new FES (FES99) in which we assimilate altimetric data in addition to tide gauge measurements. As with the three previous FESs, FES99 is based on the resolution of nonlinear barotropic shallow-water equations on a finite element grid (similar to the one used to calculate FES95.2.1) in a rotating frame. The bottom friction is parameterized by a quadratic law. The tidal forcing is the astronomical potential taking into account earth tides, self-attraction, and ocean loading tides. The two last effects are deduced from FES98 and computed by O. Francis (1999, personal communication). The application of the block resolution method used for FES98 allows us to produce solutions independent of any tide gauge and altimetric datasets. But, due to uncertainties in bathymetry, astronomical potential, or inaccuracies of the hydrodynamic equations, these "free" solutions differ significantly from in situ data measurements. In comparison with the ST95 tide gauge dataset (Andersen et al. 1995), the complex differences in amplitude and phases reach values of $\sim 40 \%$ for the semidiurnal waves and $\sim 30 \%$ for the diurnal waves. This surely results from the absence of parameterization, for example, of the internal tide dissipation in our hydrodynamic equations, which is certainly a new field to investigate in the future (Egbert and Ray 2000). Thus, to correct these discrepancies, data are assimilated in the same way as FES98 by using a representer approach (Lyard 1999). To improve the tide solutions both in the deep ocean and along the coastlines, not only tide gauge data were assimilated but also tidal information derived from T/P. Moreover, thanks to the experience gained by the computation of FES98, FES99 benefited from an assimilation procedure based upon a more realistic weighting scheme. The introduction of altimeter information in this new FES adds information on the deep ocean tides that is in agreement with what is reported in Andersen et al. (1995) and Shum et al. (1997).

In the following, the improvements yielded by FES99 are compared to former FESs. We will not go into further detail here on the hydrodynamic model; refer to the papers describing FES94 and FES95.2.1. The block resolution and the assimilation scheme are developed in the paper presenting FES98. In section 2 the T/P crossover data assimilation is fully described. In section 3 we consider the two sources of information to assimilate. The weights used for the assimilation procedure are explained for the tide gauge measurement, the altimetric analyses, and the model itself. In section 4, the quality of FES99 is evaluated, by comparing FES99 and former FESs to the tide gauge and altimetric datasets.

\section{Ocean tides from altimeter crossover data}

\section{a. Introduction}

To assimilate altimeter information into the finite element model, we chose to rely on T/P data sampled at crossover locations. By definition, crossovers are formed where ascending and descending altimeter ground tracks meet. For an individual 10-day repeat cycle the theoretical maximum is $127^{2}$ crossover locations, of which roughly $60 \%$ are over the oceans. The heritage of the crossover data type goes back to the use of altimeter observations for estimating radial orbit corrections and minimizing errors related to the altimeter timing bias. For T/P we assume that others already solve such effects and that the residual errors (other than ocean variability) are small and random, so they can be ignored in retrieving tidal constants from crossover data. We note that all usual altimeter corrections, except for the ocean and load tides and in some cases the inverse barometer (IB) correction, are applied as suggested in the documentation accompanying the geophysical data records. We exclude corrections that deal with the removal of ocean and load tides at long periodic, diurnal, and semidiurnal frequencies. The solid earth tide and Pole tide corrections are applied as provided in the geophysical data records. Exceptions in applying the altimeter corrections are discussed below. 


\section{b. Motivation for using crossover data}

In the traditional sense, crossover data comprise height difference observations at intersection locations. In this study this is not necessarily the case. Instead, we interpolate all measured data at the intersection point. The procedure results in a time series of sea level anomalies. The sampling properties compared to straightforward colinear sampling, that is, the cycle-to-cycle time series at a location that is repeated once every 10 days, is now subject to investigation. The rationale for using crossover data is related to the recovery of tidal information from altimeter data. During this recovery procedure we need to address the way T/P ground tracks are projected on the surface of the earth. Both issues are discussed in Schrama and Ray (1994), where it is explained that the main semidiurnal waves such as $\mathbf{M}_{2}$ and $S_{2}$, respectively, fold to periods of 62.1 and 58.7 days on the T/P ground track. To separate $M_{2}$ and $S_{2}$, approximately three years of data would be required. A potential weakness of the T/P system is to decouple the $\mathrm{K}_{1}$ and the $\mathrm{S}_{\mathrm{sa}}$ tides, which need a theoretical separation time of nine years. In Schrama and Ray (1994), it is also mentioned that this situation improves when use is made of crossover data providing additional samples at the same geographical location compared to the colinear sampling method. This procedure improves the least squares estimation used to recover the desired tidal constants.

\section{c. Crossover computation}

Rather than the actual observed positions, we used a nominal orbit representing the mean T/P ground track. A starting point in the procedure is a colinear stack file, which is derived from the online collection of all original geophysical data records including modifications as stored in the radar altimeter database system maintained by the Delft Institute for Earth Oriented Space research (Naeije et al. 2000).

From this database we compute the mean ground track at 1-s intervals relative to the point where the orbiter crossed the equator for all available T/P data up to cycle 236. This interpolation method is referred to as colinear interpolation. The repeat mode itself is chosen as a nominal orbit in the T/P project. It is designed as a follow-up to feasibility studies conducted prior to the launch of the system. The colinear interpolation method assumes that the satellite ground track repeats sufficiently close to replicas measured in previous 10day cycles. It does not take into account the usually negligible errors introduced by residuals in the spatial gradient of the geoid. In this case the error introduced by a residual geoid gradient is equivalent to $l d N / d s$, where $l$ equals the average deviation of the true altimeter position relative to the mean (better than $1 \mathrm{~km}$ for $\mathrm{T} /$ $\mathrm{P}$ ), while $d N / d s$ is the slope in the geoid. In extreme cases the slope term exceeds (in the absolute sense) a
10 -m-over-100-km gradient $\left(10^{-4}\right)$. In most parts of the ocean this gradient is around $10^{-5}$, so the colinear interpolation error is less than $1 \mathrm{~cm}$. The error introduced over high slope areas such as trenches or seamounts is often a factor of 10 higher. These incidental events are rejected by means of editing procedures that test for the repeatability of the measured sea level anomalies interpolated at the 1-s intervals in the colinear stack file. For this reason and within the context of this study there is no need to consider a cross-track or along-track geoid gradient correction procedure. Using the procedure described above, we find in total 7990 theoretically predicted crossovers, while in reality 7140 are retrieved from the colinear stack file. At each location in this file there is a time series consisting of sea level anomalies measured by the altimeter.

\section{d. Inverse barometer correction}

The IB correction that originates from the T/P geophysical data records (GDRs) is another source of concern. The algorithm used on the T/P GDRs relies on a local hydrostatic response equation that is computed with a constant atmospheric reference pressure of $1013.3 \mathrm{mb}$. Inputs are the 6-hourly European Centre for Medium-Range Weather Forecasts (ECMWF) global ocean mean sea surface pressure fields. There is an interaction with the semidiurnal ocean tides, and, in particular, with respect to forcing terms at the high end of the semidiurnal band. This is especially the case for the $\mathrm{S}_{2}$ tide, which is not only the result of gravitational forcing terms but also radiational terms (Chapman and Lindzen 1970). The radiational effect at the $S_{2}$ frequency can be made visible by analyzing the difference between the actual spectrum seen by a tide gauge, for instance, obtained by a direct FFT analysis, and the background spectrum modeled by a smooth admittance approach. The IB correction provided on the T/P GDRs introduces an unwanted artifact at the $S_{2}$ frequency; this type of correction should only be applied at periods of three days or longer (see also Ray and Mitchum 1996).

The obvious remedy to this artifact is to avoid the 6hourly ECMWF grids and to rely instead on daily averaged mean sea level pressure grids in the IB computation. The alternative is to apply an $S_{2}$ air pressure correction to modify the unwanted effect introduced by the standard T/P GDR IB equation. The correction itself comes in the form of a pressure correction in millibars:

$$
\mathrm{S}_{2}(\theta, t)=1.161 \sin ^{3} \theta \sin \left(2 t+159^{\circ}\right),
$$

where $t$ is the local solar time converted to appropriate units and $\theta$ is the colatitude. Equation (1) represents the main effect in the $S_{2}$ air tide that resembles a traveling wave with a phase lag relative to the sun (the theory is further explained in Chapman and Lindzen 1970). The constants in Eq. (1) are provided by R. Ray (2000, personal communication). 
Both procedures (daily averaging or application of the above equation) have been investigated within the scope of this study, and it turns out that the correction equation is equally effective in removing the unwanted effect. For most users it is logistically much easier to implement the latter option in their software than to apply daily averaged pressure grids (in their own IB model), which are hard to obtain from the ECMWF.

To summarize the $\mathrm{S}_{2}$ air tide discussion, the $\mathrm{S}_{2}$ wave modeled in the FES99 solution is corrected for the air tide leakage effect introduced by the inverted barometer correction equation on the T/P GDRs. Users that process the T/P GDRs to obtain sea level anomalies should apply the FES99 ocean and loading corrections including a pressure correction as given by Eq. (1). However, the contrary is also true. If a tidal model would have been constructed from the T/P GDRs without the application of Eq. (1), then an artifact would have been absorbed in the $S_{2}$ tide map of that model and users of sea level anomaly maps do not have to apply Eq. (1). In this case, tidal modelers would face an erroneous discrepancy between the $\mathrm{S}_{2}$ tide map of this model and in situ gauge data.

\section{e. Along-track filtering}

It should also be noted that an attempt was made to suppress short-scale spatial features in the tides caused by internal wave generation, which are visible in the observed sea level anomalies. From Ray and Mitchum (1996), it is known that the mean power in the T/P sea level anomalies as a result of internal tides is concentrated at spatial wavelengths around $160 \mathrm{~km}$. To reduce the surface elevation effect caused by such internal waves, a 20-s window is centered on each crossover position. The purpose of this data window is to select all altimeter-observed sea level anomalies that are the input for the polynomial estimation procedure. Evaluation of a four-parameter data compression polynomial at the crossover position then serves as a least squares filter to suppress the baroclinic tide-induced surface elevations.

Unfortunately, our internal wave filtering procedure also rejects crossover points in some small enclosed seas such as the Sulu Sea in the Indonesian Archipelago. The FES99 solution relies on a priori information in these regions.

\section{Choice of parameters for the assimilation}

The main purpose in computing FES99 was to overcome the uncertainties that occur in our free global tide model (i.e., without introducing any kind of in situ or remote sensing measurements) by using an assimilation procedure (Egbert et al. 1994; Egbert and Bennett 1996). As a result, FES99 is mainly improved thanks to the assimilation of tide gauge and altimetric information. The aim of an assimilation scheme is to combine the information provided by the available data (in our case the tide gauge measurements and the T/P altimetric analyses) with the information derived from a numerical model (i.e., the hydrodynamic equations solved with the finite element model). These different sets of information are optimally used to best fit the data and the dynamics in a least squares sense. The representer method is used to compute FES99 from the former free hydrodynamic solution (the a priori solution), which lacks accuracy. A representer is a field that gives the error correlation that our hydrodynamic finite element model propagates from one interpolated position of the mesh to all the other ones. One representer is associated with each assimilated data point, and has the dimensions of the tidal elevation field. This leads to the classical result of the representer method (Le Provost et al. 1998). In other words, the global solution is the sum of the a priori solution plus a weighted linear combination of the representers:

$$
\alpha=\alpha_{\text {prior }}+\sum_{k} b_{k} r_{k},
$$

where $\alpha$ is the assimilated sea surface elevation, $\alpha_{\text {prior }}$ is the a priori solution, $b_{k}$ (respectively $r_{k}$ ) is the computed vector (representer) associated with the $k$ th assimilated datum. A strong correlation between a given assimilated position and another position yields to an undamaged tide computation only if the assimilated datum is accurate. On the other hand, if the assimilated datum is not so accurate, the solutions at all the correlated positions (not only on the position associated with the representer) will also lack accuracy. Therefore, it is essential to assimilate very accurate data. As the data that can be assimilated come from various sources, their associated accuracies are too different to be considered homogeneous. The different types of information provided cannot influence the computation of a solution with the same weights. Therefore, to take into account these differences, confidence levels (inverses of errors) are set on each of the data values used in the assimilation. In practice, these confidences are the weights to apply to each representer to compute the final solution. The selection of these confidences is a key element of the assimilation scheme. Moreover, we cannot consider our model itself to be error free. Therefore, dynamic errors are included in the tidal forcing. In the following, we present the different errors introduced in our assimilation method based on a variational representer approach.

\section{a. Tide gauge data}

The assimilated tide gauge data used to produce FES99 are those used to compute FES98. They are extracted from three different databases: the World Ocean Circulation Experiment (WOCE; Ponchaut et al. 2001), the International Association for the Physical Sciences of the Ocean (IAPSO; Smithson 1992) and the Inter- 
TABLE 1. Assimilated tide gauge data as a function of the classification of each coverage type.

\begin{tabular}{lrrrrrccc}
\hline \hline Constituent & $\mathrm{M}_{2}$ & $\mathrm{~S}_{2}$ & $\mathrm{~N}_{2}$ & $\mathrm{~K}_{2}$ & $2 \mathrm{~N}_{2}$ & $\mathrm{~K}_{1}$ & $\mathrm{O}_{1}$ & $\mathrm{Q}_{1}$ \\
\hline Coast/island & 503 & 502 & 394 & 432 & 216 & 614 & 617 & 365 \\
Shelf & 91 & 90 & 86 & 73 & 6 & 104 & 105 & 100 \\
Deep ocean & 189 & 165 & 156 & 151 & 15 & 179 & 179 & 174 \\
Total & 783 & 757 & 636 & 656 & 237 & 897 & 901 & 639 \\
\hline
\end{tabular}

national Hydrographic Office (IHO; 1979) databases. Refer to the FES98 paper (Lefèvre et al. 2000) for an explanation of the selection method. However, major problems were identified in FES98 on the Patagonian shelf due to uncertain bathymetry and complex tidal phenomena in this area, where elevations can reach very high values such as $3 \mathrm{~m}$ for the $\mathrm{M}_{2}$ wave (Glorioso and Flather 1997). Then, by considering the previous work made for the local model of Glorioso and Flather (1997), a careful selection of coastal tidal data was made in order to assimilate them into our hydrodynamic model. The number of tide gauge data points assimilated is given in Table 1. Figure 1 shows the location of the selected tide gauges for the $\mathrm{M}_{2}$ wave. The distribution is nearly the same for the other waves, except for $2 \mathrm{~N}_{2}$, which is less represented in tide gauge databanks because of its lower amplitude. Figure 1 underlines the sparse density of high quality tide gauge data in the deep oceans and along some coastlines.

For each of the selected tide gauge locations, a representer is computed. In the representer approach we have to assign realistic weights on all data to be assimilated in the solution. As it is very difficult to constrain the error covariance matrix associated with in situ data, we assume that tide gauge measurements are uncorrelated. In practice this is nearly the case, as the tide gauge measurements are taken at different locations with in- dependent instruments. Then, the error covariance matrix is reduced to a diagonal matrix in which each diagonal coefficient represents the a priori assumed error associated with each assimilated data. As for the calculation of FES98, we empirically set the error for each data value by classifying these data according to depth, proximity to coasts, and origin of tide gauges. This step leads us to separate the data into three classes: coast/ island, shelf, and deep ocean. The associated weights are respectively $2.5,1$, and $0.5 \mathrm{~cm}$. Table 1 gives the number of tide gauge data values assimilated in each of the three classes. As observed in Fig. 1, the information provided by the tide gauges is mainly coastal. Indeed, due to the huge and costly logistics involved, sea level stations in the deep ocean are very sparse. However, the coastal tide gauge measurements are representative of local characteristics, while deep-ocean measurements represent the long-wavelength behavior of the ocean tides. Therefore, a balance is maintained as we need more coastal data than deep-ocean data to better represent the propagation of tides.

\section{b. Altimetric data}

As presented in section 2, the analysis of T/P measurements provides an important amount of tidal constituents located at crossover positions. Time series of sea level elevation measured by the T/P altimeter system are extracted from the colinear stack file. The harmonic analysis of these time series provides a dataset of 7140 data points supplying the amplitude and phase of the main constituents of the tidal spectrum: $\mathrm{M}_{2}, \mathrm{~S}_{2}, \mathrm{~N}_{2}, \mathrm{~K}_{2}$, $\mathrm{K}_{1}, \mathrm{O}_{1}, \mathrm{P}_{1}$, and $\mathrm{Q}_{1}$. By excluding data points in high ocean circulation variability areas, a smaller amount of 5318 data points is selected.

The assimilation scheme is based on the representer

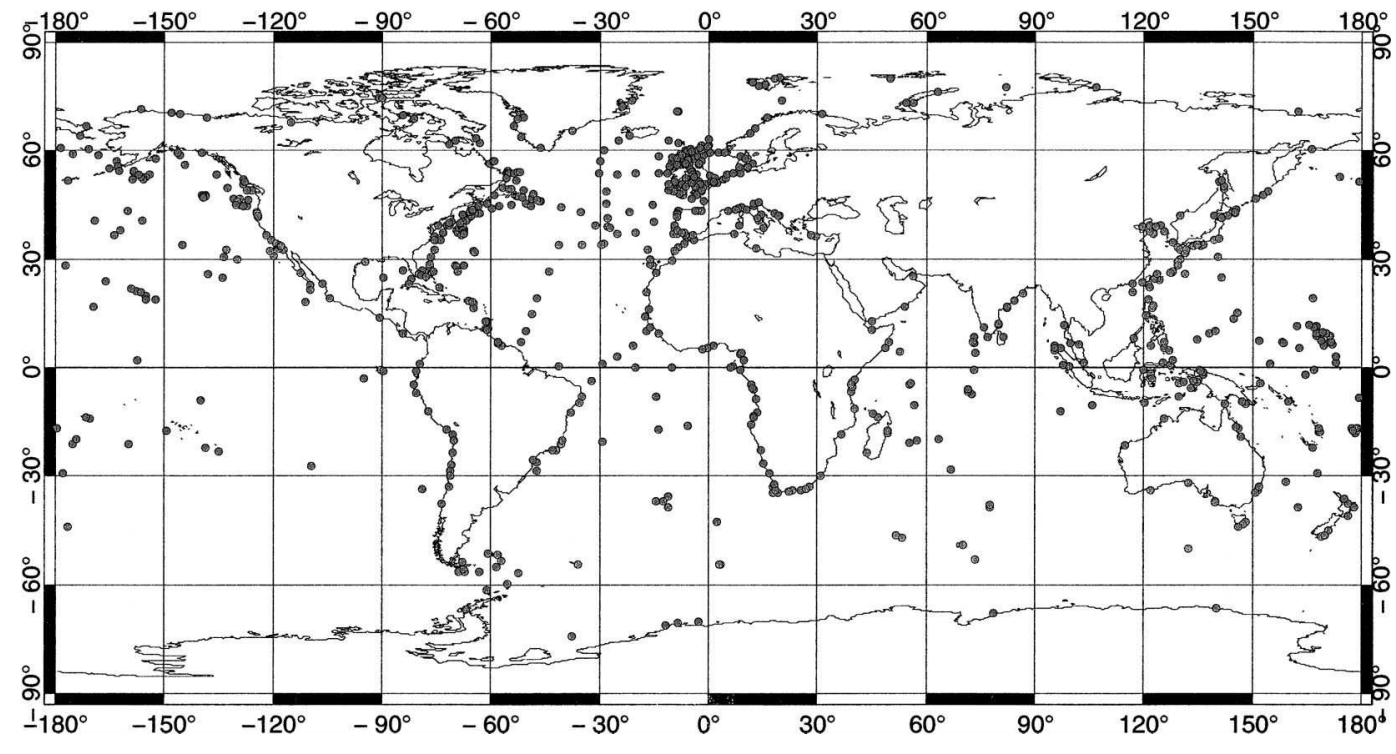

FIG. 1. Locations of the assimilated tide gauges for the $\mathrm{M}_{2}$ wave. 


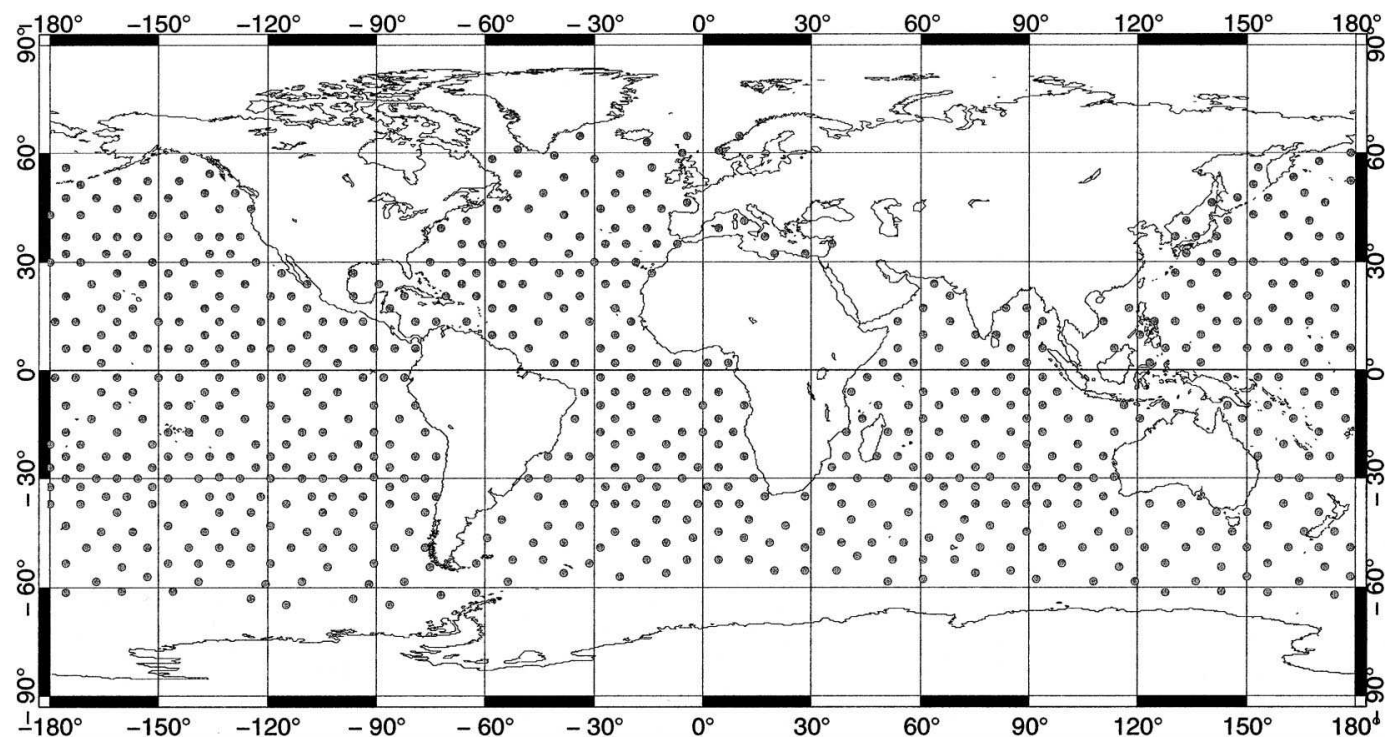

FIG. 2. Locations of the assimilated altimetric data points.

method, which intrinsically contains the dynamic behavior of our model. In the deep oceans, tide wave propagation is dominated by long wavelengths (typically $\sim 20000 \mathrm{~km}$ for the $\mathrm{M}_{2}$ wave at the equator and twice that amount for $\mathrm{K}_{1}$ ). Consequently, in these areas two representers associated with two nearby positions will contain almost the same hydrodynamic information. Unfortunately, with the present state of technology, calculation of the whole dataset of representers associated with the 5318 selected altimetric data is not practical, even on the Cray C90 supercomputers used. So, we have to make a choice between the processing capacity of the computers and the real physical contribution of each representer. For tide modeling, Egbert et al. (1994) used the method described in Bennett (1992), who showed that the computation of representers at each crossover point is not really necessary, as they are dominated by basin-scale features that are similar for nearby positions of measurement data points. They demonstrated that a full set of 6355 ocean crossovers is reduced to 986 (crossovers), which is sufficient to provide satisfactory altimetric information in their tide model using the representer method. We also select a subset of crossover data points to be assimilated by taking into account the previous results of Egbert et al. (1994), the features of our finite element meshes, and the processing capacity of the Cray computers. To extract the altimetric data for assimilation from the 5318 available data points, several criteria are applied. All the data points outside our finite element mesh are eliminated. Altimeter data points located at depths less than $200 \mathrm{~m}$ are also excluded to avoid a number of possible problems associated with the measurement process and the corrections applied (such as the tropospheric or ionospheric corrections). Moreover, as we assimilate coastal tide gauge data, we do not need to add altimetric assimilated information in shallow water. Finally, a minimal distance $(600 \mathrm{~km})$ between crossovers is set to avoid oversampling of assimilated data and excessive computation of representers. These criteria allow us to select 687 altimetric data points to assimilate. Then, for each of the seven constituents, $\mathrm{M}_{2}, \mathrm{~S}_{2}, \mathrm{~N}_{2}, \mathrm{~K}_{2}, \mathrm{~K}_{1}, \mathrm{O}_{1}$, and $\mathrm{Q}_{1}$, the 687 representers associated with the 687 data points are computed. Figure 2 shows the spatial distribution of this subset. The weight associated with the assimilated altimetric data is set to $0.75 \mathrm{~cm}$, which is approximately half the T/P measurement accuracy.

\section{c. Hydrodynamic model}

In our assimilation approach, we do not assume that our model is perfect. Indeed, different sources of error occur in global tide models (Egbert et al. 1994; Le Provost et al. 1998). The uncertainties in the model dynamics are related to the way the shallow-water equations are set up, including the way dissipation is modeled. Moreover, the choice of grid spacing, the bathymetric base model used, and the way tidal loading is treated induce a lot of uncertainties in these models. As a result, we consider a weak-constraint approach by setting dynamic errors in our model and, thus, using a variational method to obtain an optimal estimation of the global ocean tides, by combining the data with our model. We assume a uniform real Gaussian spatial shape for the error covariances, as in Le Provost et al. (1998). However, we do not assume the same dynamic errors of the hydrodynamic model for each of the different diurnal and semidiurnal constituents. In our computations, the whole set of representers is derived at the expense of computer time. Calculating the final solution by adding weighted representers to the prior solution is a relatively cheap operation. The computation of dif- 


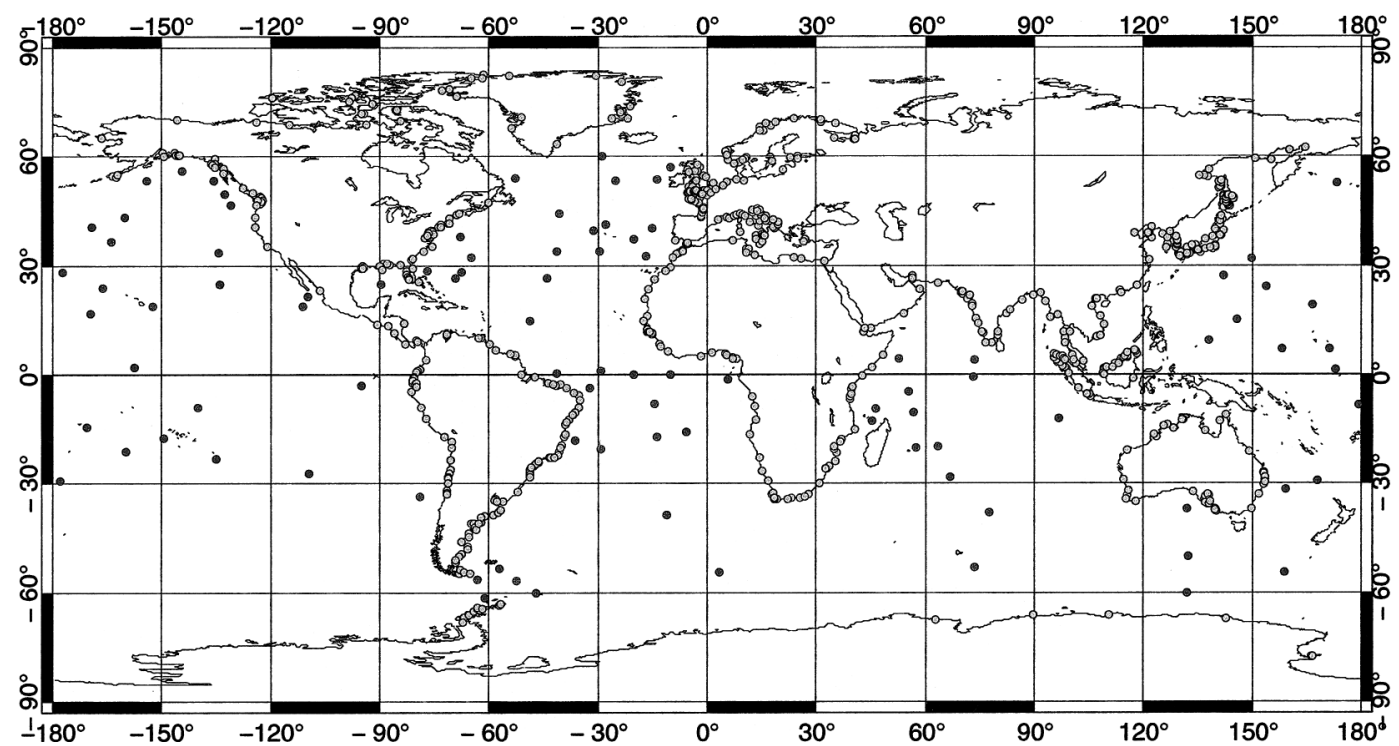

FIG. 3. Distribution of ST95 (black dots) and ST739 (open circle) comparison tidal datasets.

ferent solutions for each constituent of the tide spectrum with the same representers but different weights is practically feasible. Several successive calculations allow us to compute the error factor to apply to our forcing that which best fits both the model and the data. This is done by comparing prior and posterior solutions. Then, as opposed to FES95.2.1 and FES98, which are computed assuming a uniform dynamic variance, the dynamic errors for FES99 are tuned by a factor depending on the amplitude of the considered constituent of the tidal spectrum.

\section{Results and validation}

\section{a. Tidal spectrum of FES99}

For FES99, tide gauge and altimetric measurements are assimilated to produce individual solutions at a global scale. Eight of the main constituents of the tidal spectrum are computed: $\mathrm{M}_{2}, \mathrm{~S}_{2}, \mathrm{~N}_{2}, \mathrm{~K}_{2}, 2 \mathrm{~N}_{2}, \mathrm{~K}_{1}, \mathrm{O}_{1}$, and $\mathrm{Q}_{1}$. But one must also consider the importance of the other constituents. The eight computed constituents represent only $75.9 \%$ of the variance of the ST95 total spectrum. As ST95 is representative of deep-ocean tides, these results highlight the lack of tidal information if we do not complement the tidal spectrum with secondary waves. Thus, 19 other constituents are added to the FES99 spectrum, deduced from spline or linear admittance (Cartwright et al. 1988; Le Provost et al. 1998). Here $\mu_{2}, v_{2}, \mathrm{~L}_{2}, \lambda_{2}$, and $\mathrm{T}_{2}$ are computed from spline functions based on $\mathrm{M}_{2}, \mathrm{~N}_{2}$, and $\mathrm{K}_{2}$. The $\varepsilon_{2}$ constituent is extrapolated by linear admittance based on $2 \mathrm{~N}_{2}$ and $\mathrm{N}_{2} ; \eta_{2}$ is calculated by a linear extrapolation based on $\mathrm{M}_{2}$ and $\mathrm{K}_{2} ; \mathrm{P}_{1}$ is computed from spline functions based on $\mathrm{Q}_{1}, \mathrm{O}_{1}$, and $\mathrm{K}_{1} ; 2 \mathrm{Q}_{1}, \rho_{1}, \sigma_{1}$ are deduced by linear interpolation based on $\mathrm{Q}_{1}$ and $\mathrm{O}_{1} ; \mathrm{J}_{1}, \chi_{1}, \mathrm{M}_{11}, \mathrm{M}_{12}, \mathrm{OO}_{1}$, $\varphi_{1}, \pi_{1}$, and $\theta_{1}$ are computed from linear interpolations based on $\mathrm{O}_{1}$ and $\mathrm{K}_{1}$. All these waves are calculated at each node of the finite element mesh. In total, 27 constituents provide a full set implemented in the tidal prediction code that comes with our FES99 model.

\section{b. Tide gauge comparisons to FES models}

To evaluate the quality of FES99, we compare previous FESs with two different tidal datasets: ST95 and ST739. The latter one is the ST727 coastal tidal dataset (Lefèvre et al. 2000) complemented with 12 tide gauges along the coasts of the Patagonian shelf. Indeed, this area of strong tidal dissipation is underestimated in ST727. As mentioned above, ST95 is representative of the deep-ocean tidal propagation. ST739 is composed of coastal tide gauges distributed along the coastlines of the World Ocean, even in polar regions. The distribution of ST95 and ST739 is shown in Fig. 3. By using the root-mean square (rms) and root-sum square (rss) already introduced in the FES 98 paper, Table 2 presents the FESs intercomparison results. FES98 presents better results than FES99 due to FES98 only assimilating tide gauge data that is constrained to better fit in comparisons with deep tide gauges.

In the deep ocean (comparisons to ST95), as one would expect, the last two generations of FES are better than the first two. However, except for the $\mathrm{M}_{2}$ wave, FES98 results are slightly better than FES99 ones. This is certainly due to the assimilation of altimetric data that distorts somewhat the FES99 solution in shallow water, where the altimetric signal is not completely representative. However, the differences are very small (a few percent only). On the other hand, the improvement for $\mathrm{M}_{2}$ is a consequence of better weights applied to the 
TABLE 2. Comparisons of FESs with ST95 and ST739. Italic numbers represent the best rms or rss calculated for each tidal constituent for the four FESs. FES95.2.1 $\mathrm{K}_{2}$ and $2 \mathrm{~N}_{2}$ are taken from FES94. For ST739 we do not have the same number of measurements for each constituent because the ST739 was mainly extracted from the IHO database (International Hydrographic Office 1979), which contains inhomogeneous data.

\begin{tabular}{|c|c|c|c|c|c|c|c|c|c|c|}
\hline Database & Solution & $\mathrm{M}_{2}$ & $\mathrm{~S}_{2}$ & $\mathrm{~N}_{2}$ & $\mathrm{~K}_{2}$ & $2 \mathrm{~N}_{2}$ & $\mathrm{~K}_{1}$ & $\mathrm{O}_{1}$ & $\mathrm{Q}_{1}$ & $\operatorname{rss}(\mathrm{cm})$ \\
\hline \multirow[t]{6}{*}{ ST95 } & Data rms & 33.55 & 12.81 & 6.79 & 3.32 & 1.01 & 11.10 & 7.66 & 1.61 & - \\
\hline & No. of data values & 95 & 95 & 95 & 95 & 95 & 95 & 95 & 95 & - \\
\hline & FES94 & 2.85 & 1.57 & 0.91 & 0.48 & 0.29 & 1.19 & 1.09 & 0.28 & 3.80 \\
\hline & FES95.2.1 & 1.74 & 1.12 & 0.89 & 0.48 & 0.29 & 1.17 & 1.05 & 0.28 & 2.82 \\
\hline & FES98 & 1.51 & 0.86 & 0.80 & 0.35 & 0.31 & 1.00 & 0.93 & 0.25 & 2.41 \\
\hline & FES99 & 1.30 & 0.87 & 0.82 & 0.38 & 0.33 & 1.09 & 0.96 & 0.27 & 2.35 \\
\hline \multirow[t]{6}{*}{ ST739 } & Data rms & 60.30 & 22.22 & 12.48 & 6.58 & 1.84 & 13.47 & 9.75 & 2.01 & - \\
\hline & No. of data values & 739 & 737 & 625 & 628 & 263 & 736 & 732 & 403 & - \\
\hline & FES94 & 17.08 & 6.97 & 4.65 & 2.57 & 1.16 & 5.51 & 4.42 & 1.10 & 20.52 \\
\hline & FES95.2.1 & 24.48 & 18.97 & 5.49 & 2.57 & 1.16 & 7.07 & 4.65 & 1.09 & 32.71 \\
\hline & FES98 & 17.49 & 6.28 & 4.74 & 2.39 & 1.07 & 4.83 & 3.61 & 1.11 & 20.30 \\
\hline & FES99 & 10.58 & 5.39 & 3.95 & 2.27 & 1.03 & 4.24 & 3.26 & 1.01 & 13.87 \\
\hline
\end{tabular}

model, in particular in the adaptation of the hydrodynamic errors to each tidal constituent. Another interesting result of FES99 is that in comparison with FES94, the semidiurnal constituents are better $\left(54.4 \%\right.$ for $\mathrm{M}_{2}$ and $44.6 \%$ for $\left.\mathrm{S}_{2}\right)$ than the diurnal ones $\left(8.4 \%\right.$ for $\mathrm{K}_{1}$ and $11.9 \%$ for $\mathrm{O}_{1}$ ). This can be explained by the longer wavelength of the diurnal tides, which are thus better modeled by the original finite element model and better constrained by the dynamics included in the representers. The RSS values give an overall result of the improvement of the FESs. It can be noticed that the newer the FES, the better the RSS. The overall improvement of FES99 is $38.2 \%, 16.7 \%$, and $2.5 \%$ compared to FES94, FES95.2.1, and FES98, respectively.

Along the coasts and in shallow water (comparisons to ST739), the numerical results are very clear and show the biggest improvement in the quality of FES99. FES95.2.1 displays poor results because of spurious local resonance problems due to the assimilation procedure in shallow water. Compared to FES94, which is often considered the best global tidal model along the coasts, the improvement is $38.1 \%$ for $\mathrm{M}_{2}, 22.7 \%$ for $\mathrm{S}_{2}, 23.0 \%$ for $\mathrm{K}_{1}$, and $26.2 \%$ for $\mathrm{O}_{1}$, which underscores the real benefit of assimilating coastal measurements. Along coastlines, assimilated tidal information is mainly supplied by tide gauges. Therefore, tuning the dynamic error function of each constituent allowed us to best adapt the assimilation in shallow water. Assimilating altimetric data and adapting dynamic errors at the same time yielded major improvements in FES99 over FES98.

\section{c. Standard deviation reductions at T/P and ERS-2 crossover points}

The previous tests estimate the accuracy of the FESs wave by wave. These tests must also consider the accuracy of the new global solutions. The admittance application of relationships implies a more complete expansion of the tidal spectrum, which can be used in the prediction code. This code provides more accurate information on the quality of a tide model at the global scale. A tide model associated with a prediction code is essential for altimetric data, as of all the altimetric corrections, tides represent the largest signal to be removed in order to access the nontidal oceanic signal. So, as another tool for evaluation, we use the prediction code developed at CLS-France to study the reduction of the standard deviation in sea level from two subsets of T/P and ERS-2 measurements. As a general rule, we assumed that the lower the standard deviation in the residual tide corrected altimetric data, the better the model, since the other applied corrections are identical. TOPEX/Poseidon provides accurate measurements, while ERS-2 measurements allow independent comparison of FES95.2.1 and FES99, as these models assimilate T/P data. For T/P data, we consider cycles 11 (12 December 1992) to 259 (5 October 1999) and for ERS2, cycles 0 (29 April 1995) to 45 (9 June 1999). These cycles represent $6.8 \mathrm{yr}$ for T/P and $4.1 \mathrm{yr}$ for ERS-2. For each cycle, more than 4000 to nearly 8000 (respectively more than $26000-49000)$ crossover data points are available for T/P (respectively ERS-2) comparisons. The differences in the amount of data between different cycles are due to the numerous corrections applied before computations. The time period of altimetric observations is long enough to provide good information for the comparisons. The three FES95.2.1, FES98, and FES99 models are tested. FES94 is not included in the tests since the distributed tidal spectrum is smaller (13 waves) and predictions would lack accuracy. To test the tidal correction, O. Francis (1999, personal communication) provided ocean loading tides derived from each FES. Each loading tide solution is consistent with its associated FES to optimize the altimetric comparisons. Two tests are performed for each of the two selected satellites. Test 1 uses the global set of altimetric data. Test 2 only considers crossover points located at depths greater than $1000 \mathrm{~m}$ representing deepocean tide phenomena. The numerical results highlight the quality of FES99 (Table 3). Indeed, for each test and for each satellite, FES99 is better than FES95.2.1 
TABLE 3. Standard deviation reduction at crossover points. Test 1 gives the residual standard deviations for the whole ocean, and test 2 for bathymetries higher than $1000 \mathrm{~m}$.

\begin{tabular}{clcc}
\hline \hline Satellite & Solution & Test 1 $(\mathrm{cm})$ & Test 2 $(\mathrm{cm})$ \\
\hline T/P & FES95.2.1 & 10.70 & 7.17 \\
& FES98 & 9.60 & 7.90 \\
& FES99 & 8.54 & 6.99 \\
ERS-2 & FES95.2.1 & 15.17 & 13.06 \\
& FES98 & 14.23 & 13.26 \\
& FES99 & 13.48 & 12.65 \\
\hline
\end{tabular}

and FES98. This is mainly a consequence of the improvements introduced since FES95.2.1 and because FES98 is independent of altimetric data. Because of the shallow-water resonance modeling problem in the FES95.2.1 solution, test 1 is not too convincing for this solution (25.3\% worse than FES99). However, the quality of FES95.2.1 in deep ocean is only $2.6 \%$ worse than FES99 (test 2). An unexpected result is the quality of FES98 in the deep oceans, since it is only $13.0 \%$ worse than FES99 although no altimetric data and very few bottom tide gauges are used to compute it in these areas. However, considering the ERS-2 standard deviation reduction with FESs independent of ERS-2 measurements, the two tests again suggest that FES99 is performing better than FES95.2.1 and FES98 at the global scale (test 1) and in the deep ocean (test 2). At the global scale, FES99 is $12.5 \%$ better than FES95.2.1 and 5.6\% better than FES98. The comparative improvements in the former case are a consequence of the tide gauge data assimilation in shallow water, which improves the quality of FES98. In deep ocean, FES99 is $3.2 \%$ better than FES95.2.1 and $4.8 \%$ better than FES98. All these tests emphasize the improvement at the crossover points for FES99 compared to the former FESs.

\section{d. Standard deviation reductions at global scale}

The standard deviation reduction at the two satellites' crossovers provides an effective way to check the quality of the FESs. However, we can also test the three FESs at the global scale by making use of each alongtrack data point. For T/P, we consider cycles 95 (13 April 1995) to 195 (10 January 1998) and for ERS-2, cycles 0 (29 April 1995) to 28 (19 January 1998). These cycles are selected to represent more than $2 \mathrm{yr}(2.6 \mathrm{yr})$ of validated data over the same time period with a reasonable computation time. We should notice that the strong 1997 El Niño period is also included in our time selection. Different tests show that its influence is important at the global scale, as it contributes to a major sea level variation phenomenon. For each cycle, $\sim 4 \times$ $10^{5}$ (respectively, $\sim 14 \times 10^{5}$ ) data points are available for the T/P (ERS-2) comparisons. The huge amount of data points allows us to make a valuable quality assessment of our numerical results. As for the crossovers tests, we calculate the standard deviation of the alti-
TABLE 4. Standard deviation reduction along tracks.

\begin{tabular}{ccc}
\hline \hline Solution & T/P $(\mathrm{cm})$ & ERS-2 $(\mathrm{cm})$ \\
\hline FES95.2.1 & 11.33 & 12.38 \\
FES98 & 10.94 & 12.09 \\
FES99 & 10.66 & 11.79 \\
\hline
\end{tabular}

metric residuals computed with each of the FESs, the other GDR corrections remaining the same. These new along-track tests select FES99 as the best one to reduce the along-track standard deviation (Table 4). For T/P, FES99 provides a standard deviation $5.9 \%$ better than FES95.2.1 and 2.6\% better than FES98. For ERS-2, FES99 gives a standard deviation $4.8 \%$ better than FES95.2.1 and 2.5\% better than FES98. However, these results are global, and do not provide local information on the improvements between the three models. Figures 4 and 5 present the spatial distribution of the rms reductions of sea level altimetric residuals of FES99 compared to the two other FES tide corrections. Deeper blue in the map corresponds to lower sea level standard deviation computed with FES99, and better FES99 compared to the other FES. The differences are mainly located around $0 \mathrm{~cm}$ (between $\pm 0.05 \mathrm{~cm}$ ), which is very small compared to the order of $10 \mathrm{~cm}$ given by Table 4. However, a few high-variation areas are underlined by these figures. The differences between FES99 and FES95.2.1 show that in deep ocean the two solutions are virtually identical. The main differences occur along the coasts and on the continental shelves. The standard deviation computed with FES99 is lower than with FES95.2.1 in several local areas. The areas where the standard deviation is lower by $1 \mathrm{~cm}$ (deep blue) are the following: the Sri Lanka strait, the eastern part of the Andaman Sea, the Gulf of Tonkin, the East China Sea, the Yellow Sea, the Sulu Sea, the Celebes Sea, the Timor Sea, the main part of the Australian coastlines, the eastern part of the Okhotsk Sea, the eastern part of the Bering Sea, the Gulf of California, the Patagonian shelf, the Denmark Strait, the Norwegian Sea, the Irish Sea, and the eastern and western part of the North Sea. Yet there remain areas with a standard deviation higher than $1 \mathrm{~cm}$ (red) for FES99: the southern part of the Barents Sea, the area between the islands of the Philippines, a small western part of the Okhotsk Sea and the Bering Sea, the mouth of the Amazon, and the Hudson Bay. However, these results are not conclusive since the altimetric analyses in such complex and coastal areas as the Philippines and the Amazon are not accurate. For the Barents Sea we do not have any explanations for the differences. In the Hudson Bay, FES95.2.1 is based on FES94, which is very good in this bay, as the solution is computed with open boundary conditions set by in situ measurements. On the other hand, no tide gauge observations and very little altimetric information are assimilated in FES99 in this bay. The overall FES99 achieves its principal purpose compared to FES95.2.1: to provide an improved global tide solution in shallow 


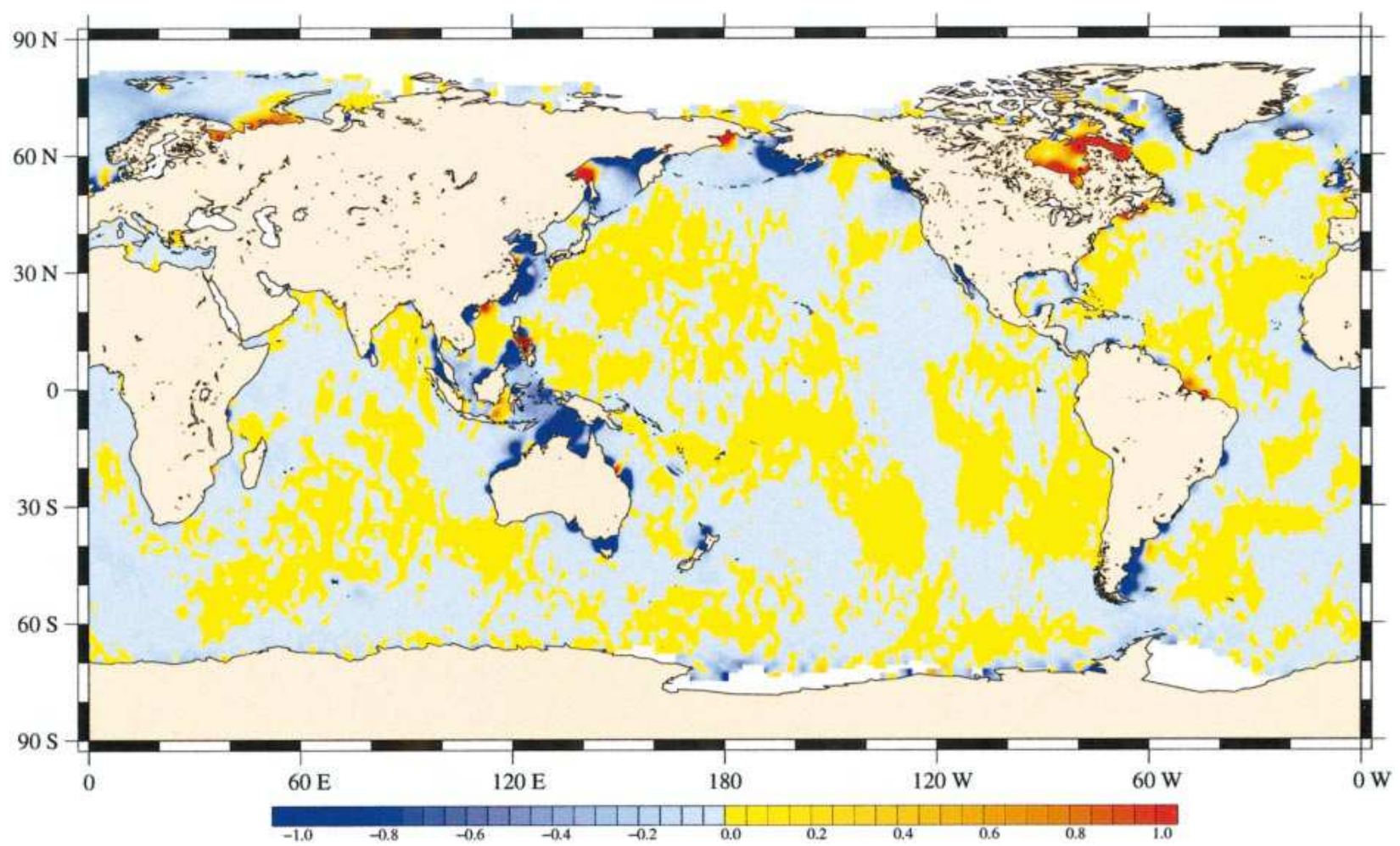

FIG. 4. The rms differences of sea level anomalies between FES99 and FES95.2.1. (Units are in $\mathrm{cm}$.)

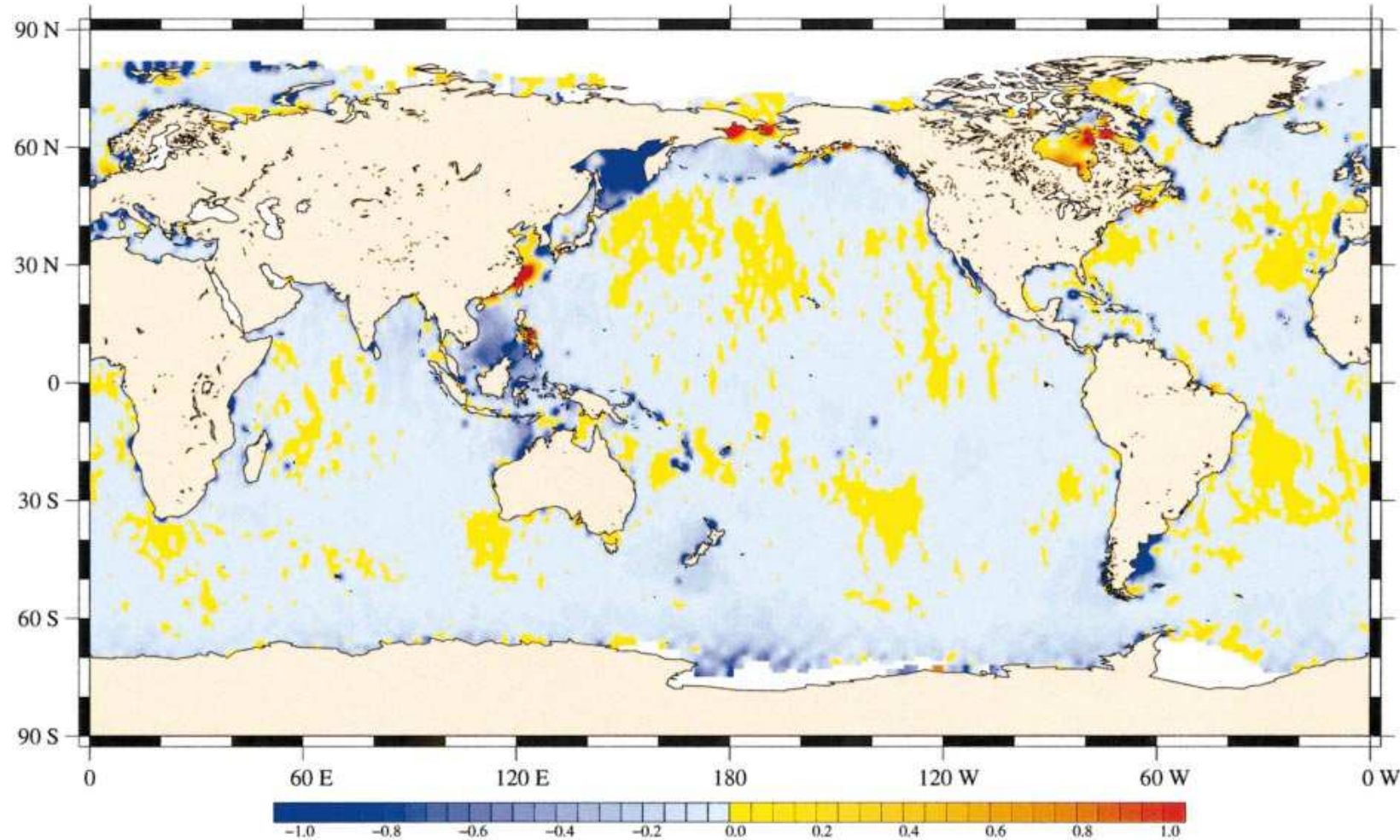

FIG. 5. The rms differences of sea level anomalies between FES99 and FES98. (Units are in cm.) 
waters, continental shelves, and semienclosed seas. The comparison of the sea level variance deduced with FES99 and FES98 leads to better results for FES99, except in the East China Sea and the Bering Sea. Globally the sea level anomaly standard deviation calculated with FES99, taken as an altimetric correction among the numerous altimetric corrections, is better than the one computed with FES98. However, contrary to FES95.2.1, the differences are smoother, which is certainly due to the use of an assimilation scheme similar to FES98 in shallow water (assimilation of tide gauge measurements). Indeed, no altimetric data in depths shallower than $200 \mathrm{~m}$ are used in the FES99 assimilation procedure.

\section{Conclusions}

By assimilating complementary tidal information from in situ (selected tide gauge) and altimetric (subset of $\mathrm{T} / \mathrm{P}$ crossovers) data points in our hydrodynamic model, we have been able to produce a new global tide solution, FES99, which is a major improvement on previous FESs. The computation of tidal solutions completely independent of any kind of measurement does not provide sufficiently accurate results for the traditional applications of tide models. This is why we apply a specific assimilation procedure based on the representer approach to eliminate the uncertainties of our hydrodynamic model (bathymetry, friction, modeling of internal tides, etc.). The combination of tide gauge and $\mathrm{T} / \mathrm{P}$ altimeter-derived data in our assimilation improves the solutions both in the deep ocean and along coastlines. As we have already used the specific assimilation scheme in a finite element approach (using tide gauges) to produce FES98, FES99 benefits from a better understanding and a better handling of the assimilation procedure (application of new weights). The introduction of T/P crossover altimetric information in FES99 adds tidal signal information in the deep ocean. The crossover computation method used to provide altimetric harmonic data to assimilate at specific points is original. The source file is a collection of modified geophysical data records maintained by the Delft Institute for Earth Oriented Space research, as well as the harmonic decomposition code and the daily filtering of the IB correction. The quality of FES99, underlined by comparisons to tide gauge datasets and residual variance at T/P and ERS-2 crossovers and along tracks, demonstrates the value of our hydrodynamic model combined with a representer approach.

The principal aim of FES99 is to provide the scientific and engineering communities with a new, improved FES model compared to our previous solution, called FES95.2.1. This goal has been accomplished, as shown by the numerical comparisons in this study. Indeed, for the eight main constituents of the tidal spectrum, the FES99 rms differences with the ST95 open ocean are now only $1.30 \mathrm{~cm}$ for $\mathrm{M}_{2}$ (respectively $1.74 \mathrm{~cm}$ for
FES95.2.1) and $1.09 \mathrm{~cm}$ for $\mathrm{K}_{1}(1.17 \mathrm{~cm})$. On the basis of ST95, the computed rss of the differences between observations and solutions is reduced from $2.82 \mathrm{~cm}$ for FES95.2.1 to $2.35 \mathrm{~cm}$ for FES99 (an improvement of $16.6 \%)$. The comparisons with the coastline dataset ST739 highlight the real improvement of FES99. The computed RSS of $20.52 \mathrm{~cm}$ for FES94 (considered the best hydrodynamic model along coastlines) is reduced to $13.87 \mathrm{~cm}$ for FES99 (an improvement of $32.4 \%$ ). However, as FES99 assimilated tide gauge and T/P data, we also compared our FESs to ERS-2 measurements, which are completely independent. Using a similar prediction code, we computed crossover and along-track residual standard deviations. In the deep ocean (where FES95.2.1 local resonance effects do not occur), the residual standard deviation is reduced from $13.06 \mathrm{~cm}$ for FES95.2.1 to $12.65 \mathrm{~cm}$ for FES99 (an improvement of $3.1 \%$ ). Along track, it is reduced from 12.38 to 11.79 $\mathrm{cm}$ (an improvement of $4.8 \%$ ).

However, it should be noticed that our hydrodynamic model still lacks accuracy in shallow-water regions and continental shelves. A future investigation would be to introduce new parameters in our tidal equations such as the internal waves or the baroclinic tidal effects.

These new FES99 solutions are available on a $0.25^{\circ}$ $\times 0.25^{\circ}$ gridded version of the full finite element solutions with its associated prediction code and its derived ocean load tide. The FES99 tide model package can be supplied on request from the lead author.

Acknowledgments. FES computations were run on the Cray computer at the Institut du Développement et des Ressources en Informatique Scientifique (IDRIS). The altimetric comparison tests were performed at CLS (France), which provided computers and algorithms for tidal prediction. The authors would like to thank Joël Dorandeu and Michaël Ablain from CLS for their support. We are grateful to Philip Woodworth from the Proudman Oceanographic Laboratory, Bidston Observatory, Birkenhead, United Kingdom, for his useful comments.

\section{REFERENCES}

Andersen, O. B., 1995: Global ocean tides from ERS-1 and TOPEX/ Poseidon. J. Geophys. Res., 100, 25 249-25 259.

— - P. L. Woodworth, and R. A. Flather, 1995: Intercomparison of recent ocean tide models. J. Geophys. Res., 100, 25 261-25 282.

Bennett, A. F, 1992: Inverse methods in physical oceanography. Monograph on Mechanics and Applied Mathematics, Cambridge Monogr., Cambridge University Press, 364 pp.

Cartwright, D. E., R. Spencer, J. M. Vassie, and P. L. Woodworth, 1988: The tides of the Atlantic Ocean. Philos. Trans. Roy. Soc. London, 324A, 513-563.

Chapman, S., and R. R. Lindzen, 1970: Atmospheric Tides, Thermal and Gravitational. D. Reidel, 200 pp.

Eanes, R. J., and S. V. Bettadpur, 1994: Ocean tides from two years of TOPEX/POSEIDON altimetry. Eos, Trans. Amer. Geophys. Union, 75 (Suppl.), 61.

— for Space Research Tech. Memo. CSR-TM-95-06. 
Egbert, G. D., and A. F. Bennett, 1996: Data assimilation methods for ocean tides. Modern Approaches to Data assimilation in Ocean Modeling, P. Malanotte-Rizzoli, Ed., Elsevier Science, 147-179.

— , and R. D. Ray, 2000: Significant dissipation of tidal energy in the deep ocean inferred from satellite altimeter data. Nature, 405, 775-778.

, A. F. Bennett, and M. G. G. Foreman, 1994: TOPEX/POSEIDON tides estimated using a global inverse model. J. Geophys. Res., 99, 24 821-24 852.

Glorioso, P. D., and R. A. Flather, 1997: The Patagonian Shelf tides. Progress in Oceanography, Vol. 40, Pergamon, 263-293.

International Hydrographic Office, 1979: Tidal Constituent Bank Station Catalogue. Ocean and Aquatic Sciences, Department of Fisheries and Oceans, Ottawa, Canada.

Lefèvre, F., F. H. Lyard, and C. Le Provost, 2000: FES98: A new global tide finite element solution independent of altimetry. Geophys. Res. Lett., 27, 2717-2720.

Le Provost, C., M. L. Genco, F. Lyard, P. Vincent, and P. Canceil, 1994: Spectroscopy of the world ocean tides from a finite element hydrodynamic model. J. Geophys. Res., 99, 24 777-24 797.

, F. Lyard, J. M. Molines, M. L. Genco, and F. Rabilloud, 1998 A hydrodynamic ocean tide model improved by assimilating a satellite altimeter-derived data set. J. Geophys. Res., 103, 55135529.

Lyard, F. H., 1999: Data assimilation in a wave equation: A variational representer approach for the Grenoble tidal model. J. Comput. Phys., 149, 1-31.

— , and M. L. Genco, 1994: Optimization methods for bathymetry and open boundary conditions in a finite element model of ocean tides. J. Comput. Phys., 114, 234-256.

Naeije, M., E. J. O. Schrama, and R. Scharroo, 2000: The Radar Altimeter Database System project RADS. Proc. Int. Geoscience and Remote Sensing Symp., Honolulu, HI, IEEE, 487-490.

Ponchaut, F., F. Lyard, and C. Le Provost, 2001: An analysis of the tidal signal in the WOCE sea level dataset. J. Atmos. Oceanic Technnol., 18, 77-91.

Ray, R., 1999: A global ocean tide model from TOPEX/Poseidon Altimetry/GOT99.2. NASA Tech. Memo 209478, 58 pp.

_ - and G. T. Mitchum, 1996: Surface manifestation of internal tides generated near Hawaii. Geophys. Res. Lett., 23, 2101-2104

Schrama, E. J. O., and R. D. Ray, 1994: A preliminary tidal analysis of TOPEX/Poseidon altimetry. J. Geophys. Res., 99, 24 799-24 808.

Shum, C. K., and Coauthors, 1997: Accuracy assessment of recent ocean tide models. J. Geophys. Res., 102, 25 173-25 194.

Smithson, M. J., 1992: Pelagic Tidal Constants. Vol. 35. IAPSO Publication Scientifique, $191 \mathrm{pp}$ 